\title{
ROJ
}

\section{Prognostic significance of lymphovascular invasion in patients with prostate cancer treated with postoperative radiotherapy}

\author{
Jae-Uk Jeong ${ }^{1}$, Taek-Keun Nam¹, Ju-Young Song ${ }^{1}$, Mee Sun Yoon', Sung-Ja Ahn', Woong-Ki Chung ${ }^{1}$, Ick Joon \\ Cho', Yong-Hyub Kim', Shin Haeng Cho', Seung II Jung ${ }^{2}$, Dong Deuk Kwon ${ }^{2}$ \\ 'Department of Radiation Oncology, Chonnam National University Hwasun Hospital, Chonnam National University Medical \\ School, Hwasun, Korea \\ ${ }^{2}$ Department of Urology, Chonnam National University Hwasun Hospital, Chonnam National University Medical School, Hwasun, Korea
}

Purpose: To determine prognostic significance of lymphovascular invasion (LVI) in prostate cancer patients who underwent adjuvant or salvage postoperative radiotherapy (PORT) after radical prostatectomy (RP)

Materials and Methods: A total of 168 patients with prostate cancer received PORT after RP, with a follow-up of $\geq 12$ months. Biochemical failure after PORT was defined as prostate-specific antigen (PSA) $\geq 0.2 \mathrm{ng} / \mathrm{mL}$ after PORT or initiation of androgen deprivation therapy (ADT) for increasing PSA levels regardless of the value. We analyzed the clinical outcomes including survivals, failure patterns, and prognostic factors affecting the outcomes.

Results: In total, 120 patients (71.4\%) received salvage PORT after PSA levels were $>0.2 \mathrm{ng} / \mathrm{mL}$ or owing to clinical failure. The 5 -year biochemical failure-free survival (BCFFS), clinical failure-free survival (CFFS), distant metastasis-free survival (DMFS), overall survival, and cause-specific survival rates were 78.3\%, 94.3\%, 95.0\%, 95.8\%, and 97.3\%, respectively, during a follow-up range of 12-157 months (median: 64 months) after PORT. On multivariate analysis, PSA level of $\leq 1.0 \mathrm{ng} / \mathrm{mL}$ at the time of receiving PORT predicted favorable BCFFS, CFFS, and DMFS. LVI predicted worse CFFS $(p=0.004)$ and DMFS $(p=0.015)$. Concurrent and/or adjuvant ADT resulted in favorable prognosis for BCFFS $(p<0.001)$ and CFFS $(p=0.017)$.

Conclusion: For patients with adverse pathologic findings, PORT should be initiated as early as possible after continence recovery after RP. Even after administering PORT, LVI was an unfavorable predictive factor, and further intensive adjuvant therapy should be considered for these patients.

Keywords: Prostate neoplasms, Prostatectomy, Postoperative radiotherapy

\section{Introduction}

Radical prostatectomy (RP) is a therapeutic option for patients with prostate cancer. However, recurrence is frequently observed in patients with high-risk features in surgical specimens such as positive surgical margin (PSM) or extracapsular extension (ECE) [1,2]. Moreover, the incidence of recurrence and metastasis is generally high in patients who

Received 18 June 2019, Revised 16 July 2019, Accepted 14 August 2019.

Correspondence: Taek-Keun Nam, Department of Radiation Oncology, Chonnam National University Hwasun Hospital, Chonnam National University Medical School, 322 Seoyang-ro, Hwasun 58128, Korea. Tel: +82-61-379-7200, Fax: +82-61379-7249, E-mail: tknam@jnu.ac.kr (http://orcid.org/0000-0003-1229-8468)

(c) This is an Open Access article distributed under the terms of the Creative Commons Attribution Non-Commercial License (http://creativecommons.org/ licenses/by-nc/4.0/) which permits unrestricted non-commercial use, distribution, and reproduction in any medium, provided the original work is properly cited.

www.e-roj.org 
experience biochemical failure (BCF) after RP [3]. Therefore, adjuvant treatment should be considered for patients with a high risk of recurrence after RP. In fact, adjuvant radiation therapy (RT) can reduce the risk of recurrence in such patients [4]. Furthermore, several randomized studies of adjuvant RT versus the wait-and-see approach after RP strongly suggested the use of adjuvant RT immediately rather than waiting when the level of prostate-specific antigen (PSA) increases in highrisk patients [5-7].

Although the incidence of prostate cancer is lower in Korea than in western countries, there has been a rapid increase in the prevalence. By 2017, prostate cancer was the third most common cancer (304.1 per 100,000 Korean men). However, the 5-year survival rate increased from 55.9\% during 1993-1995 to $93.9 \%$ during 2012-2016 in Korea [8,9]. Moreover, most Korean hospitals recommended the use of postoperative radiation therapy (PORT) in patients with high-risk features after RP [10]. However, most clinical data about PORT have been obtained in western countries, and there are only a few reports about the results in Korea. Therefore, we aimed to analyze the treatment results of PORT performed at a single institution and evaluated the associated prognostic factors.

\section{Materials and Methods}

\section{Patients}

Patients with prostate cancer who received PORT after RP between January 2006 and December 2015 were included in this study. The inclusion criteria were as follows: patients who received PORT after RP for histologically confirmed localized adenocarcinoma of the prostate and follow-up duration of $\geq 12$ months. The exclusion criteria were patients who experienced distant metastases before PORT, those who had received prior pelvic radiotherapy or previous chemotherapy, and those with a history of another malignancy. All patients underwent routine examinations including a digital rectal examination and serum PSA measurements every 3 months during the first 3 years, every 6 months over the next 2 years, and annually thereafter. Imaging workups-including computed tomography, magnetic resonance imaging, whole body bone scintigraphy, and positron emission tomography/ computed tomography-were performed in patients with suspected recurrence during the follow-up period. This study was approved by the Institutional Review Board at Chonnam National University Hwasun Hospital (No. CNUHH-2019-111).

\section{Treatments}

Patients with prostate cancer were asked to choose between RP and RT in the Department of Urology. Patients who underwent RP received PORT as adjuvant or salvage treatment if indicated. Adjuvant RT was defined as RT delivered to patients with adverse pathologic features such as ECE, PSM, or seminal vesicle invasion even when PSA levels were $\leq 0.2 \mathrm{ng} /$ $\mathrm{mL}$ after RP. Salvage RT was defined as RT delivered when PSA levels were $>0.2 \mathrm{ng} / \mathrm{mL}$ at the start of RT or owing to clinical failure. The clinical target volume (CTV) was confined to the surgical bed only in patients with no evidence of pelvic lymph node involvement or those with a probability of pelvic lymph node involvement less than 20\% according to the Roach formula, 2/3×PSA+(Gleason score-6)×10 [11].

If the probability of pelvic lymph node involvement was more than $20 \%$ or pelvic lymph node involvement was confirmed in surgical specimens, CTV was whole pelvis including the surgical bed, and the internal iliac, external iliac, and common iliac lymph nodes. The CTV was expanded to form planning target volume in all directions by $8 \mathrm{~mm}$ except $5 \mathrm{~mm}$ posteriorly for CTV of prostate fossa. The RT techniques used for patients were intensity-modulated RT (IMRT), threedimensional-conformal RT (3D-CRT), and a combination of 3D-CRT and IMRT. The simultaneous integrated boost technique was used for the surgical bed when the fraction exceeded 2.0 Gy, while 1.8 or 2.0 Gy per fraction was administered to the pelvic nodal region. For bladder and rectum preparation, each patient was instructed to empty bladder and rectum, and then drink two cups of water 1 hour before acquisition of planning $\mathrm{CT}$ and each treatment. For verification of patient's position, daily electronic portal image via 6D ExacTrac (BrainLAB, Feldkirchen, Germany) and weekly cone beam CT were obtained in Novalis Tx (Varian Medical Systems, Palo Alto, CA, USA), or daily megavoltage CT was obtained in helical tomotherapy. Dose constraints for rectum and bladder were as follows: $V_{66}$ $<1 \mathrm{~mL}$ and $\mathrm{V}_{35}<40 \%$ for rectum; $\mathrm{V}_{68}<1 \mathrm{~mL}$ and $\mathrm{V}_{45}<40 \%$ for bladder. To adjust for various dose fractionations, the total equivalent dose $\left(\mathrm{EQD}_{2 / 2}\right)$ was calculated in 2-Gy fractions for prostate cancer $(\alpha / \beta$ ratio $=2.0)$. Most patients received androgen deprivation therapy (ADT). Neoadjuvant ADT (NA-ADT) was ADT administered prior to 1 month before RT. Concurrent ADT was ADT administered within 1 month before RT and at least 1 month during RT. Adjuvant ADT was ADT maintained for at least 12 months after RT.

\section{Statistical analysis}

Biochemical failure-frees survival (BCFFS) was the duration between the first date of PORT and the date of serum PSA 
Table 1. Patients and treatment characteristics

\begin{tabular}{|c|c|}
\hline Characteristic & Value \\
\hline Age (yr) & $68(50-78)$ \\
\hline \multicolumn{2}{|l|}{ Surgery type } \\
\hline Laparoscopic & $79(47.0)$ \\
\hline Robotic & $53(31.6)$ \\
\hline Retropubic & 33 (19.6) \\
\hline Transperineal & $3(1.8)$ \\
\hline \multicolumn{2}{|l|}{ Pathologic T stage } \\
\hline $1-2$ & $54(32.2)$ \\
\hline $3 a$ & $65(38.7)$ \\
\hline $3 b-4$ & $46(27.3)$ \\
\hline Unknown & $3(1.8)$ \\
\hline \multicolumn{2}{|l|}{ Pathologic N stage } \\
\hline 0 & $159(94.6)$ \\
\hline 1 & $9(5.4)$ \\
\hline \multicolumn{2}{|c|}{ Postoperative Gleason score } \\
\hline$\leq 6$ & $10(6.0)$ \\
\hline 7 & $72(42.8)$ \\
\hline$\geq 8$ & $82(48.8)$ \\
\hline Unknown & $4(2.4)$ \\
\hline \multicolumn{2}{|l|}{ Resection margin } \\
\hline Negative & $89(53.0)$ \\
\hline Positive & $79(47.0)$ \\
\hline \multicolumn{2}{|l|}{ Lymphovascular invasion } \\
\hline No & $143(85.1)$ \\
\hline Yes & $25(14.9)$ \\
\hline \multicolumn{2}{|l|}{ Perineural invasion } \\
\hline No & $39(23.2)$ \\
\hline Yes & $129(76.8)$ \\
\hline \multicolumn{2}{|l|}{ PSA level (ng/mL) } \\
\hline Initial & $14.930(2.140-177.000)$ \\
\hline At PORT (ng/mL) & $0.275(0.001-97.184)$ \\
\hline \multicolumn{2}{|l|}{ Treatment setting } \\
\hline Adjuvant & $48(28.6)$ \\
\hline Salvage & $120(71.4)$ \\
\hline RT dose & $67.2(60.0-72.6)$ \\
\hline $\mathrm{RT}$ dose, $\mathrm{EQD}_{2 / 2}$ & $69.3(60.0-76.2)$ \\
\hline \multicolumn{2}{|l|}{ RT volume } \\
\hline Surgical bed only & $46(27.4)$ \\
\hline Whole pelvis & $122(72.6)$ \\
\hline \multicolumn{2}{|l|}{ RT method } \\
\hline IMRT & $121(72.0)$ \\
\hline 3D-CRT & $24(14.3)$ \\
\hline Mixed & $23(13.7)$ \\
\hline \multicolumn{2}{|l|}{ NA-ADT } \\
\hline No & 113 (67.3) \\
\hline Yes & $55(32.7)$ \\
\hline \multicolumn{2}{|l|}{ CA-ADT } \\
\hline No or unknown & $41(24.4)$ \\
\hline Yes & $127(75.6)$ \\
\hline
\end{tabular}

Values are presented as median (range) or number (\%). PSA, prostate-specific antigen; PORT, postoperative radiotherapy; $\mathrm{RT}$, radiotherapy; $\mathrm{EQD}_{2 / 2}$, equivalent dose in 2 Gy fractions at $\alpha /$ $\beta$ ratio of 2.0; IMRT, intensity-modulated radiotherapy; 3D-CRT, three-dimensional conformal radiotherapy; NA-ADT, neoadjuvant androgen deprivation therapy before PORT; CA-ADT, concurrent or adjuvant androgen deprivation therapy with PORT. level of $\geq 0.2 \mathrm{ng} / \mathrm{mL}$ according to the Nara definition, which was similar to the BCF definition after RP [12]. Clinical failurefree survival (CFFS) was the duration between the first date of PORT and the date of disease progression including local failure, regional failure, and distant metastasis on imaging or histology. Distant metastasis-free survival (DMFS) was the duration between the first date of PORT and the date of metastases to distant organs or non-regional lymph nodes on imaging or histology. Overall survival (OS) was the duration between the first date of PORT and the date of the patient's death or censored to the final date of follow-up. Cancerspecific survival (CSS) was the interval between the first date of PORT and the date of death from progressive prostate cancer or treatment complications. Radiation-related toxicity was evaluated according to the Common Terminology Criteria for Adverse Events (version 5.0). Kaplan-Meier models were used for survival analysis of all potential factors that affected treatment results and were tested by using the log-rank test. A Cox proportional hazards model was used for multivariate analysis. A chi-squared test was used to determine the treatment factors related to toxicity. On statistical analysis, p-values less than 0.05 were considered significant. All statistical analyses were performed using SPSS version 19.0 (IBM SPSS, Armonk, NY, USA).

\section{Results}

\section{Patients and treatment characteristics}

A total of 168 patients were eligible in this study. The patient and treatment characteristics are summarized in Table 1. The time interval between RP and PORT ranged from 1 to 19 months (median, 4) in the adjuvant aim and from 1 to 128 months (median, 14) in the salvage aim. In all patients, the median preoperative PSA level and PSA level at the time of PORT were $14.930 \mathrm{ng} / \mathrm{mL}$ (range, 2.140 to $177.000 \mathrm{ng} / \mathrm{mL}$ ) and $0.275 \mathrm{ng} / \mathrm{mL}$ (range, 0.001 to $97.184 \mathrm{ng} / \mathrm{mL}$ ), respectively. In total, 120 patients (71.4\%) received salvage RT. Of the 120 patients, 109 received RT owing to elevated PSA levels $>0.2 \mathrm{ng} / \mathrm{mL}$ without clinical failure and 11 patients owing to locoregional clinical failure. In patients with adjuvant and salvage RT, the median PSA level at the time of RT were 0.009 $\mathrm{ng} / \mathrm{mL}$ (range, 0.001 to $0.200 \mathrm{ng} / \mathrm{mL}$ ) and $0.546 \mathrm{ng} / \mathrm{mL}$ (range, 0.001 to $97.184 \mathrm{ng} / \mathrm{mL}$ ), respectively. The radiation field was confined to the surgical bed only in 46 patients and to the whole pelvis in 122. The dose fractionation schedule consisted of 1.8 or 2.0 Gy fractions ( $=99$ ), 2.1 or 2.2 Gy fractions ( $=62)$, or mixed fractions of $2.0-2.5 \mathrm{~Gy}(n=7)$. The nominal 


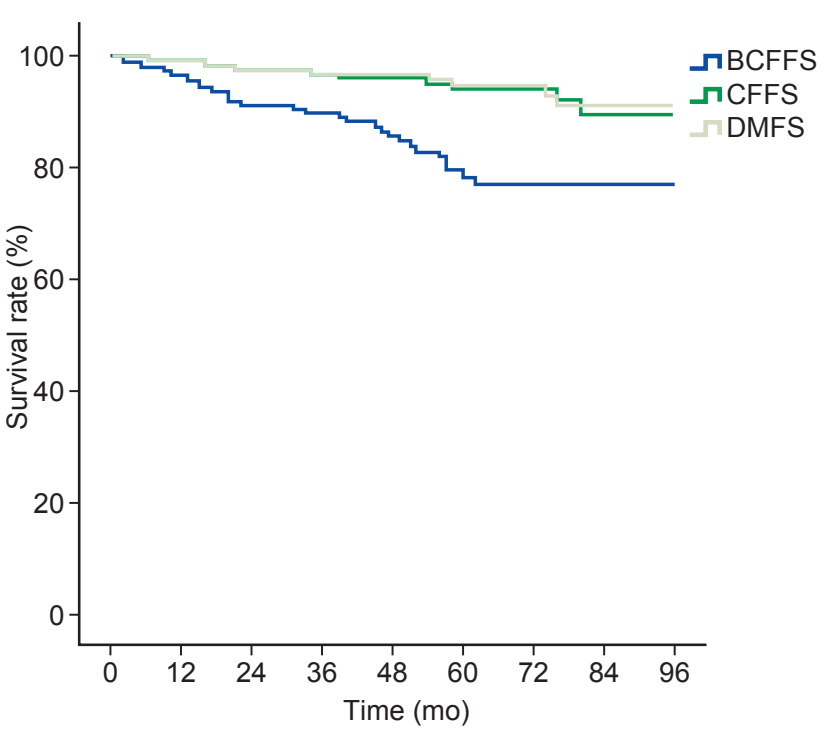

Fig. 1. Biochemical failure-frees survival (BCFFS), clinical failurefree survival (CFFS), and distant metastasis-free survival (DMFS).

dose of RT ranged from 61.6 to $76.0 \mathrm{~Gy}$ (median, 66.0) in the adjuvant aim and from 60.0 to $72.6 \mathrm{~Gy}$ (median, 68.0) in the salvage aim. The median duration of NA-ADT was 4 months (range, 1 to 66 months) and the median duration of concurrent and/or adjuvant ADT (CA-ADT) was 17 months (range, 1 to 70 months). Of 55 patients who received NA-ADT, 46 patients received CA-ADT while 9 did not. Among 127 patients who received CA-ADT, 24 patients (14.3\%) received concurrent ADT only, 14 (8.3\%) received adjuvant ADT only, and 89 (53.0\%) received both.

\section{Treatment outcomes and pattern of failure}

The median follow-up duration was 64 months (range, 12 to 157 months). Overall, the estimated 5-year BCFFS, CFFS, DMFS, OS, and CSS rates were 78.3\%, 94.3\%, 95.0\%, 95.8\%, and $97.3 \%$, respectively (Fig. 1). During the follow-up period, BCF was observed in 31 patients (18.4\%) and clinical failure in $11(6.5 \%)$. Among these 11 patients with clinical failure, 2 patients (1.1\%) experienced locoregional failure alone, 5 (3.0\%) showed distant metastasis alone, and the remaining 4 (2.4\%) experienced both. Among 25 patients with lymphovascular invasion (LVI), 5 patients (20\%) developed clinical failure: 4 patients developed distant metastasis and 1 patient developed both regional and distant failure. In 143 patients without $L V I$, only 6 patients (4.2\%) developed clinical failure. Four patients $(2.4 \%)$ died due to progression of prostate cancer among the 11 patients (6.5\%) who died during the follow-up period. Among 55 patients who received NA-ADT, the BCFFS
(87.6\% vs. $18.8 \% ; p<0.001)$ and OS $(96.6 \%$ vs. $60 \% ; p=$ 0.011 ) were significantly higher in the 46 patients treated with CA-ADT than in the 9 patients treated without CA-ADT. The treatment outcomes were not significantly different among the subgroups of patients who received concurrent ADT only, those who received adjuvant ADT only, and those who received both. Among patients who received salvage RT, the treatment outcomes of the 109 patients with persistently elevated PSA without clinical failure did not differ significantly from those of the remaining 11 patients with clinical failure.

\section{Prognostic factors related to treatment outcomes}

The results for univariate analysis of prognostic factors are shown in Table 2. Among these factors, the Gleason score and PSA level at the time of RT were significant factors commonly affecting BCFFS, CFFS, and DMFS. The results of multivariate analysis using above 15 variates are shown in Table 3. The PSA level $>1.0 \mathrm{~mL}$ at the time of PORT was a significant factor for BCFFS, CFFS, and DMFS. Among the pathologic features, the presence of LVI was an adverse prognostic factor for CFFS and DMFS (Fig. 2). NA-ADT was an adverse prognostic factor for BCFFS and CFFS. CA-ADT was a favorable prognostic factor for BCFFS and CFFS.

\section{Treatment-related toxicity}

Treatment-related toxicity was acceptable during the followup period (Table 4). After a median of 25.5 months after PORT completion (range, 1 to 78 months), grade 2 or 3 genitourinary (GU) toxicities developed in 40 patients. In addition, 5 patients developed grade 2 or 3 gastrointestinal (GI) toxicities after a median of 29.4 months (range, 10 to 60 months) after PORT completion. Grade $3 \mathrm{GU}$ toxicity was mostly gross hematuria requiring intervention. Three patients (1.8\%) reported grade 2 Gl toxicity of hematochezia, and 2 patients (1.2\%) had grade 3 toxicity with rectal bleeding, requiring endoscopic coagulation. Three patients had both GU and GI toxicities of grade 2 or 3. There was a significant relationship between $\mathrm{EQD}_{2 / 2}$ and complications. Among 42 patients with grade 2 or 3 toxicities, 30 patients received PORT with an EQD $2 / 2$ dose of $\geq 69.3 \mathrm{~Gy}$ and 12 patients received PORT with an $\mathrm{EOD}_{2 / 2}$ dose of $<69.3 \mathrm{~Gy}$ ( $\mathrm{p}$ $=0.002$ ). Of 30 patients who received an $\mathrm{EQD}_{2 / 2}$ dose of $\geq 69.3$ Gy, 19 (63.3\%) received PORT with fractions of more than 2 Gy. Among surgery-related complications at the last follow-up, urinary incontinence remained in 63 patients (37.5\%). Erectile dysfunction was observed in 135 patients (80.4\%), unknown in 27 patients (16.1\%), and the function was preserved in the remaining 6 patients (3.5\%). 
Table 2. Univariate analysis of prognostic factors for 5 -year survivals

\begin{tabular}{|c|c|c|c|c|c|c|c|}
\hline \multirow{2}{*}{ Variable } & \multirow{2}{*}{$\begin{array}{c}\text { No. of } \\
\text { patients }\end{array}$} & \multicolumn{2}{|c|}{ BCFFS } & \multicolumn{2}{|c|}{ CFFS } & \multicolumn{2}{|c|}{ DMFS } \\
\hline & & $\%$ & p-value & $\%$ & p-value & $\%$ & $p$-value \\
\hline Age (yr) & & & 0.517 & & 0.669 & & 0.236 \\
\hline$\leq 67$ & 82 & 77.3 & & 92.0 & & 92.0 & \\
\hline$>67$ & 86 & 79.1 & & 96.3 & & 97.6 & \\
\hline Initial PSA (ng/mL) & & & 0.102 & & 0.069 & & 0.218 \\
\hline$\leq 10$ & 52 & 86.7 & & 95.9 & & 98.0 & \\
\hline $10-20$ & 53 & 78.3 & & 98.1 & & 98.1 & \\
\hline$>20$ & 63 & 71.2 & & 89.8 & & 89.8 & \\
\hline Gleason score & & & 0.046 & & 0.045 & & 0.032 \\
\hline$\leq 7$ & 82 & 87.6 & & 98.8 & & 98.8 & \\
\hline $8-10$ & 82 & 68.6 & & 90.7 & & 92.2 & \\
\hline pT stage & & & 0.073 & & 0.231 & & 0.227 \\
\hline $1-2$ & 54 & 82.8 & & 97.9 & & 100 & \\
\hline $3 a$ & 65 & 82.0 & & 96.0 & & 96.0 & \\
\hline 3b-T4 & 46 & 68.1 & & 88.9 & & 88.9 & \\
\hline pN stage & & & 0.010 & & 0.060 & & 0.353 \\
\hline 0 & 159 & 80.4 & & 94.9 & & 95.7 & \\
\hline 1 & 9 & 37.5 & & 80.0 & & 80.0 & \\
\hline Resection margin & & & 0.468 & & 0.366 & & 0.149 \\
\hline Negative & 89 & 78.8 & & 96.4 & & 97.7 & \\
\hline Positive & 79 & 78.4 & & 91.5 & & 91.5 & \\
\hline PNI & & & 0.478 & & 0.927 & & 0.829 \\
\hline No & 39 & 88.6 & & 93.8 & & 93.8 & \\
\hline Yes & 129 & 74.9 & & 94.6 & & 95.5 & \\
\hline LVI & & & 0.300 & & 0.001 & & $<0.001$ \\
\hline No & 143 & 79.8 & & 96.6 & & 97.4 & \\
\hline Yes & 25 & 71.1 & & 79.5 & & 79.5 & \\
\hline PSA at PORT (ng/mL) & & & $<0.001$ & & $<0.001$ & & $<0.001$ \\
\hline$\leq 0.2$ & 73 & 96.6 & & 97.8 & & 97.8 & \\
\hline$>0.2$ and $\leq 0.5$ & 33 & 83.2 & & 100 & & 100 & \\
\hline$>0.5$ and $\leq 1.0$ & 24 & 78.4 & & 100 & & 100 & \\
\hline$>1.0$ & 38 & 37.0 & & 78.7 & & 81.8 & \\
\hline Treatment aim & & & 0.001 & & 0.222 & & 0.300 \\
\hline Adjuvant & 48 & 97.0 & & 96.8 & & 96.8 & \\
\hline Salvage & 120 & 70.8 & & 93.3 & & 94.3 & \\
\hline $\mathrm{EQD}_{2 / 2}(\mathrm{~Gy})$ & & & 0.569 & & 0.254 & & 0.214 \\
\hline$<69.3$ & 82 & 74.3 & & 97.3 & & 97.3 & \\
\hline$\geq 69.3$ & 86 & 80.4 & & 92.3 & & 93.6 & \\
\hline RT volume & & & 0.254 & & 0.182 & & 0.265 \\
\hline Surgical bed & 46 & 88.6 & & 96.2 & & 96.2 & \\
\hline Whole pelvis & 122 & 74.4 & & 93.5 & & 94.5 & \\
\hline RT method & & & 0.007 & & 0.265 & & 0.306 \\
\hline IMRT & 121 & 81.2 & & 93.1 & & 94.2 & \\
\hline 3D-CRT & 24 & 59.8 & & 91.7 & & 91.7 & \\
\hline Combination & 23 & 89.5 & & 100 & & 100 & \\
\hline NA-ADT & & & 0.231 & & 0.009 & & 0.018 \\
\hline No & 113 & 79.2 & & 97.3 & & 97.3 & \\
\hline Yes & 55 & 76.9 & & 87.2 & & 89.6 & \\
\hline CA-ADT & & & $<0.001$ & & 0.037 & & 0.187 \\
\hline No or unknown & 41 & 39.4 & & 86.6 & & 86.6 & \\
\hline Yes & 127 & 89.2 & & 96.6 & & 97.5 & \\
\hline
\end{tabular}

BCFFS, biochemical failure-free survival; CFFS, clinical failure-free survival; DMFS, distant metastasis-free survival; PSA, prostatespecific antigen; PNI, perineural invasion; LVI, lymphovascular invasion; PORT, postoperative radiotherapy; EQD $2 / 2$, equivalent dose in 2 Gy fractions at $\alpha / \beta$ ratio of 2.0; RT, radiotherapy; IMRT, intensity-modulated radiotherapy; 3D-CRT, three-dimensional conformal radiotherapy; NA-ADT, neoadjuvant androgen deprivation therapy before PORT; CA-ADT, concurrent or adjuvant androgen deprivation therapy with PORT. 
Table 3. Multivariate analysis of prognostic factors for survivals

\begin{tabular}{|c|c|c|c|c|c|c|}
\hline \multirow{2}{*}{ Variable } & \multicolumn{2}{|l|}{ BCFFS } & \multicolumn{2}{|l|}{ CFFS } & \multicolumn{2}{|l|}{ DMFS } \\
\hline & $\mathrm{HR}(95 \% \mathrm{Cl})$ & $p$-value & HR (95\% Cl) & $p$-value & $\mathrm{HR}(95 \% \mathrm{Cl})$ & $p$-value \\
\hline \multicolumn{7}{|l|}{ LVI } \\
\hline No & Ref & & Ref & & Ref & \\
\hline Yes & - & NS & $9.73(2.06-45.87)$ & 0.004 & $5.96(1.41-25.22)$ & 0.015 \\
\hline \multicolumn{7}{|l|}{ pN-stage } \\
\hline No & Ref & & Ref & & Ref & \\
\hline Yes & $8.74(2.38-32.14)$ & 0.001 & - & NS & - & NS \\
\hline \multicolumn{7}{|l|}{ PNI } \\
\hline No & Ref & & Ref & & Ref & \\
\hline Yes & $11.86(3.03-46.39)$ & $<0.001$ & - & NS & - & NS \\
\hline \multicolumn{7}{|l|}{ PSA at PORT (ng/mL) } \\
\hline$\leq 0.2$ & Ref & & Ref & & Ref & \\
\hline $0.2-0.5$ & - & NS & - & NS & - & NS \\
\hline $0.5-1.0$ & - & NS & - & NS & - & NS \\
\hline$>1.0$ & $32.19(5.82-177.85)$ & $<0.001$ & $19.62(2.17-176.76)$ & 0.008 & $10.46(1.27-85.82)$ & 0.029 \\
\hline \multicolumn{7}{|l|}{ NA-ADT } \\
\hline No & Ref & & Ref & & Ref & \\
\hline Yes & $7.72(2.81-21.25)$ & $<0.001$ & $11.10(2.44-50.37)$ & 0.002 & - & NS \\
\hline \multicolumn{7}{|l|}{ CA-ADT } \\
\hline No or unknown & Ref & & Ref & & Ref & \\
\hline Yes & $0.05(0.02-0.15)$ & $<0.001$ & $0.14(0.02-0.70)$ & 0.017 & - & NS \\
\hline
\end{tabular}

BCFFS, biochemical failure-free survival; CFFS, clinical failure-free survival; DMFS, distant metastasis-free survival; HR, hazard ratio; $\mathrm{Cl}$, confidence interval; LVI, Iymphovascular invasion; PNI, perineural invasion; PSA, prostate-specific antigen; PORT, postoperative radiotherapy; NA-ADT, neoadjuvant androgen deprivation therapy before PORT; CA-ADT, concurrent or adjuvant androgen deprivation therapy with PORT.

A

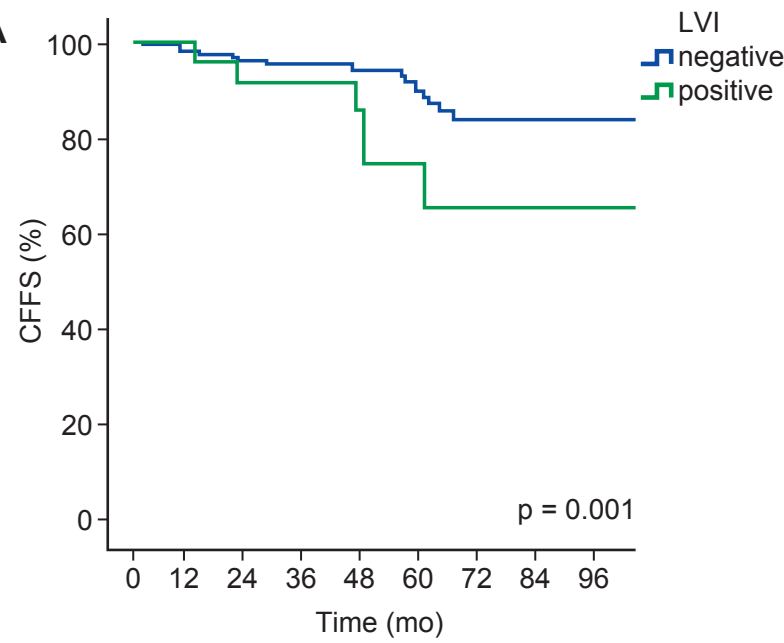

B

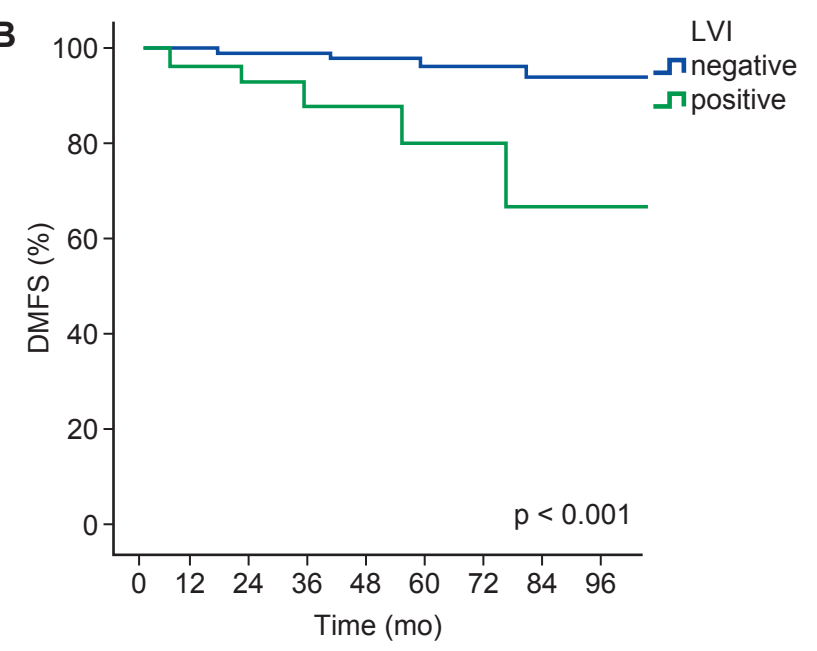

Fig. 2. (A) Clinical failure-free survival (CFFS) and (B) distant metastasis-free survival (DMFS) according to lymphovascular invasion (LVI) status.

\section{Discussion and Conclusion}

The application of adjuvant RT may reduce the probability of recurrence in patients, and the clinical benefit of adding adjuvant RT after RP has been confirmed in several randomized trials [5-7]. PORT is also effective as a salvage treatment $[13,14]$. Immediate adjuvant $\mathrm{RT}$ after $\mathrm{RP}$, compared with salvage $\mathrm{RT}$, was associated with improved BCFFS, DMFS, and OS rates in patients with ECE or PSM [15]. In the present clinical settings, 
Table 4. Treatment-related complications

\begin{tabular}{lccccc}
\hline \multicolumn{1}{c}{ Complication } & Grade 0 & Grade 1 & Grade 2 & Grade 3 & Grade 4 \\
\hline Genitourinary $^{\text {a) }}$ & $115(68.5)$ & $13(7.7)$ & $34(20.2)$ & $6(3.6)$ & $0(0.0)$ \\
Gastrointestinal $^{a)}$ & $163(97.0)$ & $0(0.0)$ & $3(1.8)$ & $2(1.2)$ & $0(0.0)$ \\
\hline
\end{tabular}

Values are presented as number of patients (\%).

a) Evaluated by Common Terminology Criteria for Adverse Events version 5.0.

immediate adjuvant RT is not generally administered to high-risk patients because of older age and the presence of comorbidities $[16,17]$. Nevertheless, PORT including salvage RT resulted in excellent treatment outcomes: both the 5-year OS and CSS rates were more than 90\% $[13,18]$. In the present study, the use of PORT resulted in 5-year BCFFS, CFFS, DMFS, OS, and CCS rates of 78.3\%, 94.3\%, 95.0\%, 95.8\%, and 97.3\%, respectively. These outcomes obtained in the present study were similar to those obtained in the previous studies.

Among pathologic features, one study showed that LVI was observed in $12.2 \%$ of patients who received RP [19]. The presence of $L V I$ is an unfavorable factor associated with poor treatment results [20-24]. In patients who underwent $\mathrm{RP}, \mathrm{BCFFS}$ was worse in patients with $\mathrm{LVI}$ compared to those without LVI (30\% vs. 92\%; $p=0.001)$ [23]. In a systematic review, patients with $\mathrm{LVI}$ had a higher probability for BCF after $\mathrm{RP}$ (hazard ratio $[\mathrm{HR}]=2.05 ; 95 \%$ confidence interval $[\mathrm{Cl}]$, 1.64-2.56; $p<0.001$ ) [19]. Currently, only a few studies have evaluated the prognostic significance of LVI in patients treated with PORT. LVI is a poor prognostic factor for BCFFS in patients who received salvage RT after RP (45\% vs. 100\% at 3 years; $p=0.0169$ ) [20]. In a retrospective study of 160 patients who underwent PORT after RP, LVI was a significant prognostic factor for BCFFS [25]. The 5-year BCFFS rate was 27.8\% for patients with $\mathrm{LVI}$ compared to $71.2 \%$ for those without LVI ( $p$ < 0.001). In 18 patients with LVI, the median RT dose was 60.9 Gy and only 1 patient received ADT with PORT. In the present study, the 5-year BCFFS rate of patients with LVI (71.1\%) was better than those observed in previous studies, because most patients with LVI (21 of 25 patients) received ADT with PORT and the dose of RT was higher than those reported in the other studies. In the present study, LVI was not significantly associated with BCFFS. This might be because CA-ADT could have reduced the significance of LVI for BCFFS. However, the presence of LVI maintained its significance for CFFS and DMFS, suggesting that patients with LVI had more potential to develop clinical failure after RT regardless of using CA-ADT.

The PSA level at the time of PORT can be an independent factor associated with treatment results. The BCFFS and DMFS were observed to decrease with increasing levels of PSA at the time of PORT [26]. The 5-year BCFFS rate was 71\% for patients with PSA levels of $0.01-0.2 \mathrm{ng} / \mathrm{mL}, 63 \%$ for those with PSA levels of $0.21-0.50 \mathrm{ng} / \mathrm{mL}, 54 \%$ for those with PSA levels of 0.51-1.0 ng/mL, 43\% for those with PSA levels of 1.01-2.0 $\mathrm{ng} / \mathrm{mL}$, and $37 \%$ for those with PSA levels of $>2.0 \mathrm{ng} / \mathrm{mL}$ ( $p$ $<0.001)$. Other studies also proved that the PSA level at the time of PORT was an independent prognostic factor for BCFFS $[18,27]$. Thus, early PORT when the patient has low levels of PSA could improve the treatment results of patients who underwent RP. On multivariate analysis in the present study, the PSA level $>1.0 \mathrm{ng} / \mathrm{mL}$ at the time of PORT was significantly associated with worse BCFFS, CFFS, and DMFS. There was no prognostic relevance for OS or CSS in patients with PSA levels of at the time of PORT.

The addition of ADT to PORT could be beneficial after RP. The results of a randomized trial, RTOG 9601, advocated the use of CA-ADT for patients who received postoperative salvage RT [28]. The 12-year OS (76.3\% vs. 71.3\%; $p=0.04$ ) and CSS (94.2\% vs. 86.6\%; $p<0.001)$ were higher in the ADT group than in the placebo group. Another randomized trial GETUG 16, also demonstrated the superiority of adding CAADT to postoperative salvage RT [29]. The 5-year BCFFS was significantly different between the ADT and placebo groups (80\% vs. 62\%; $p<0.001$ ). No additional complications of adding ADT were reported in both studies. In the present study, the 5-year BCFFS was also more favorable in patients who received CA-ADT (89.2\% vs. 39.4\%; $p<0.001)$. Currently, the clinical benefit of adding CA-ADT to PORT is obvious, but the optimal timing of ADT and the duration remain unknown when PORT is administered. Two ongoing randomized clinical trials, RADICALS and GETUG-17, are expected to confirm the efficacy of adding ADT to PORT. In the present study, the treatment outcomes of patients receiving NA-ADT were worse compared to those of patients not receiving NA-ADT. Of the 55 patients who received NA-ADT, ADT was administered to 39 patients (71\%) owing to biochemical failure of PSA $>0.2 \mathrm{ng} / \mathrm{mL}$ or clinical failure. This might have resulted in the delay in starting PORT and negatively affecting the results of PORT.

Generally, the radiation doses of 60-64 Gy for adjuvant RT and 66-70 Gy for salvage RT are commonly used after RP with 
conventional fraction sizes. In our institution, $\mathrm{EQD}_{2 / 2}$ ranges from 61.5 to $70.0 \mathrm{~Gy}$ in the adjuvant setting and from 60 to $76.2 \mathrm{~Gy}$ in the salvage setting. Increasing the $\mathrm{EQD}_{2 / 2}$ to $>66 \mathrm{~Gy}$ during postoperative salvage RT was significantly associated with improved $\mathrm{BCFFS}(\mathrm{HR}=0.81 ; 95 \% \mathrm{Cl}, 0.71-0.91 ; \mathrm{p}=0.001)$ [26]. In a meta-analysis of dose-response of postoperative salvage RT, the EOD $2 / 2$ of 70 Gy was significantly associated with a BCFFS rate of $58.4 \%$ compared to $38.5 \%$ for a dose of $60 \mathrm{~Gy}$ [30]. However, the present study showed that $\mathrm{EQD}_{2 / 2}$ $\geq 69.3 \mathrm{~Gy}$ was not a significant factor for clinical outcomes, which might be owing to using CA-ADT in most patients. An ongoing randomized trial of patients undergoing RP assigned to receive $\mathrm{EQD}_{2 / 2}$ of either 64 or $70 \mathrm{~Gy}$ is expected to reveal the role of dose escalation [31].

Some urologists have advocated waiting to administer PORT until the PSA level increases rather than immediately performing adjuvant RT because of concerns about toxicities. The PORT is often delayed until PSA is rising because it can adversely affect recovery of urinary continence after surgery. However, the risk of toxicities was not significantly increased in the PORT group compared with the observation group $[5,7]$. In a randomized trial that compared EQD $2 / 2$ of $64 \mathrm{~Gy}$ and $70 \mathrm{~Gy}$ for salvage RT after RP, the incidence of acute grade 2 and 3 $\mathrm{GU}$ or $\mathrm{GI}$ toxicities was similar between the groups [31]. Acute grade 2 or $3 \mathrm{GU}$ complications were reported in 13.6\% of patients treated with $64 \mathrm{~Gy}$ and in $18.3 \%$ of patients treated with $70 \mathrm{~Gy}(\mathrm{p}=0.2)$. Acute grade 2 or $3 \mathrm{Gl}$ complications were reported in 16.6\% of patients treated with $64 \mathrm{~Gy}$ and in 17.7\% of patients treated with $70 \mathrm{~Gy}(p=0.8)$. Patients receiving $>70$ Gy had clinically related worsening urinary symptoms. In a retrospective study, the risk of grade $\geq 2$ complications increased when radiation $\mathrm{EQD}_{2 / 2}$ doses exceeded $72 \mathrm{~Gy}$ during PORT (19\% vs. $6 \% ; p=0.007$ ) [32]. In the present study, a higher radiation dose was associated with treatment-related toxicities, but mostly with $>2$ Gy fractions. Therefore, we recommend that $\leq 2$ Gy fractions should be used during PORT when the radiation dose is to be increased.

The present study has several limitations. The primary limitation is the retrospective design and inherent selection bias associated with patient heterogeneity. Moreover, we could not obtain information regarding the administration of CAADT in 6 patients (3.5\%). Nevertheless, the number of those patients is small and adding CA-ADT was an independent prognostic factor for BCFFS and CFFS.

In conclusion, for patients with adverse pathologic findings, PORT should be initiated as early as possible after continence recovery after RP. Even after administering PORT, LVI was an unfavorable predictive factor in the present study, and further intensive adjuvant therapy should be considered for these patients.

\section{Conflict of Interest}

No potential conflict of interest relevant to this article was reported.

\section{References}

1. Wieder JA, Soloway MS. Incidence, etiology, location, prevention and treatment of positive surgical margins after radical prostatectomy for prostate cancer. J Urol 1998;160:299-315.

2. Epstein JI, Partin AW, Sauvageot J, Walsh PC. Prediction of progression following radical prostatectomy. A multivariate analysis of 721 men with long-term follow-up. Am J Surg Pathol 1996;20:286-92

3. Pound CR, Partin AW, Eisenberger MA, Chan DW, Pearson JD, Walsh PC. Natural history of progression after PSA elevation following radical prostatectomy. JAMA 1999;281:1591-7.

4. Swanson GP, Hussey MA, Tangen CM, et al. Predominant treatment failure in postprostatectomy patients is local: analysis of patterns of treatment failure in SWOG 8794. J Clin Oncol 2007;25:2225-9.

5. Bolla $M$, van Poppel $H$, Collette $L$, et al. Postoperative radiotherapy after radical prostatectomy: a randomized controlled trial (EORTC trial 22911). Lancet 2005;366:572-8.

6. Thompson IM Jr, Tangen CM, Paradelo J, et al. Adjuvant radiotherapy for pathologically advanced prostate cancer: a randomized clinical trial. JAMA 2006;296:2329-35.

7. Wiegel $T$, Bottke $D$, Steiner $U$, et al. Phase III postoperative adjuvant radiotherapy after radical prostatectomy compared with radical prostatectomy alone in pT3 prostate cancer with postoperative undetectable prostate-specific antigen: ARO 96-02/AUO AP 09/95. J Clin Oncol 2009;27:2924-30.

8. Jung KW, Won YJ, Kong HJ, Lee ES. Cancer statistics in Korea: incidence, mortality, survival, and prevalence in 2016. Cancer Res Treat 2019;51:417-30.

9. Park J, Suh B, Shin DW, Hong JH, Ahn H. Changing patterns of primary treatment in Korean men with prostate cancer over 10 years: a nationwide population based study. Cancer Res Treat 2016;48:899-906.

10. Chang $A R$, Park W. Radiotherapy in prostate cancer treatment: results of the patterns of care study in Korea. Radiat Oncol J 2017;35:25-31. 
11. Rahman S, Cosmatos H, Dave G, Williams S, Tome M. Predicting pelvic lymph node involvement in current-era prostate cancer. Int J Radiat Oncol Biol Phys 2012;82:906-10.

12. Miyake M, Tanaka N, Asakawa I, et al. The impact of the definition of biochemical recurrence following salvage radiotherapy on outcomes and prognostication in patients with recurrent prostate cancer after radical prostatectomy: a comparative study of three definitions. Prostate Int 2019;7:4753.

13. Trock BJ, Han M, Freedland SJ, et al. Prostate cancer-specific survival following salvage radiotherapy vs observation in men with biochemical recurrence after radical prostatectomy. JAMA 2008;299:2760-9.

14. Cotter SE, Chen MH, Moul JW, et al. Salvage radiation in men after prostate-specific antigen failure and the risk of death. Cancer 2011;117:3925-32.

15. Hwang WL, Tendulkar RD, Niemierko A, et al. Comparison between adjuvant and early-salvage postprostatectomy radiotherapy for prostate cancer with adverse pathological features. JAMA Oncol 2018;4:e175230.

16. Mahal BA, Hoffman KE, Efstathiou JA, Nguyen PL. National trends in the recommendation of radiotherapy after prostatectomy for prostate cancer before and after the reporting of a survival benefit in March 2009. Clin Genitourin Cancer 2015;13:e167-72.

17. Kalbasi A, Swisher-McClure S, Mitra N, et al. Low rates of adjuvant radiation in patients with nonmetastatic prostate cancer with high-risk pathologic features. Cancer 2014;120:3089-96.

18. Stish BJ, Pisansky TM, Harmsen WS, et al. Improved metastasis-free and survival outcomes with early salvage radiotherapy in men with detectable prostate-specific antigen after prostatectomy for prostate cancer. J Clin Oncol 2016;34:3864-71.

19. Huang $Y$, Huang $H$, Pan XW, et al. The prognostic value of Iymphovascular invasion in radical prostatectomy: a systematic review and meta-analysis. Asian J Androl 2016;18:780-5.

20. Shariat SF, Khoddami SM, Saboorian H, et al. Lymphovascular invasion is a pathological feature of biologically aggressive disease in patients treated with radical prostatectomy. J Urol 2004;171:1122-7.

21. Herman CM, Wilcox GE, Kattan MW, Scardino PT, Wheeler TM. Lymphovascular invasion as a predictor of disease progression in prostate cancer. Am J Surg Pathol 2000;24:859-63.
22. McNeal JE, Yemoto CE. Significance of demonstrable vascular space invasion for the progression of prostatic adenocarcinoma. Am J Surg Pathol 1996;20:1351-60.

23. de la Taille A, Rubin MA, Buttyan $R$, et al. Is microvascular invasion on radical prostatectomy specimens a useful predictor of PSA recurrence for prostate cancer patients? Eur Urol 2000;38:79-84.

24. van den Ouden $D$, Kranse $R$, Hop WC, van der Kwast $T H$, Schroder $\mathrm{FH}$. Microvascular invasion in prostate cancer: prognostic significance in patients treated by radical prostatectomy for clinically localized carcinoma. Urol Int 1998;60:17-24.

25. Brooks JP, Albert PS, O'Connell J, McLeod DG, Poggi MM. Lymphovascular invasion in prostate cancer: prognostic significance in patients treated with radiotherapy after radical prostatectomy. Cancer 2006;106:1521-6.

26. Tendulkar RD, Agrawal S, Gao T, et al. Contemporary update of a multi-institutional predictive nomogram for salvage radiotherapy after radical prostatectomy. J Clin Oncol 2016;34:3648-54.

27. Briganti A, Karnes RJ, Joniau S, et al. Prediction of outcome following early salvage radiotherapy among patients with biochemical recurrence after radical prostatectomy. Eur Urol 2014;66:479-86.

28. Shipley WU, Seiferheld W, Lukka HR, et al. Radiation with or without antiandrogen therapy in recurrent prostate cancer. $\mathrm{N}$ Engl J Med 2017;376:417-28.

29. Carrie C, Hasbini A, de Laroche G, et al. Salvage radiotherapy with or without short-term hormone therapy for rising prostate-specific antigen concentration after radical prostatectomy (GETUG-AFU 16): a randomised, multicentre, open-label phase 3 trial. Lancet Oncol 2016;17:747-56.

30. King CR. The dose-response of salvage radiotherapy following radical prostatectomy: a systematic review and meta-analysis. Radiother Oncol 2016;121:199-203.

31. Ghadjar P, Hayoz S, Bernhard J, et al. Acute toxicity and quality of life after dose-intensified salvage radiation therapy for biochemically recurrent prostate cancer after prostatectomy: first results of the randomized trial SAKK 09/10. J Clin Oncol 2015;33:4158-66.

32. Cozzarini C, Fiorino C, Da Pozzo LF, et al. Clinical factors predicting late severe urinary toxicity after postoperative radiotherapy for prostate carcinoma: a single-institute analysis of 742 patients. Int J Radiat Oncol Biol Phys 2012;82:191-9. 